\title{
ZU WOLFRAM VON ESCHENBACH.
}

Grimm, Gr. IV, 133 bespricht die ellipse des verbum substantivum, wenn demselben ein adjectiv und dativ der person folgt. Die ellipse wird aus dem ahd. und as. belegt; die mhd. beispiele gehören vorwiegend Wolfram an und werden nach dem Lachmann'schen texte aufgefuhrt. Grimm fährt dann fort: 'Zuweilen steht aber auch das verb. subst. ausgedrtlckt: ahd. thaz lâz thir wesan suazi $0 . \mathrm{I}, 1,41 ;$ ni lazet iu iz wesan suâr II, 16, 40; latz iu von mir niht swcere sin Parz. 555, 7 nach den handschriften.' Demnach gewinnt es den anschein, als ob an den ubrigen stellen bei Wolfram - lat iu niht leit P. 24,18. laz dir min laster leit P. 159,2. daz er im lieze ir laster leit P. 526, 28. daz lat iu durch die frounen leit P. 535, 22. nu lâ dirz durch uns bêde leit P. 689, 30. nâ lâ dir von mir niht so gâch Wh. 122,2. la dir die schrift unmore Tit. 164,4 - die handschriften die ellipse gewährleisten. Das ist aber keineswegs der fall. Die uberlieferung ist vielmehr folgende:

P. 24, 18 durch iwer zuht lat iu niht wesen (sin g) leit $\mathrm{Dg}$. durch iver zuht si iu niht leil Ggg.

Nur dg haben lat iu niht leit, dann aber z. 19 Sein das ich g. Vor abe ich d. Das Vor abe in d statt ob ist augenscheinlich verderbt.

P. 159, 2 und laz dir sin min laster leit Ddg.

La dir G. La dir min laster wesen leit $\mathrm{g}$.

P.526,28 daz er im lieze ir laster leit

29. $\operatorname{Sin} \mathrm{D}$.

daz er im lieze sin ir laster leit d. daz er im lieze ir laster sin leit $\mathrm{g}$. Er lieze im sin ir laster leit $\mathbf{G}$.

Daz im nere ir laster (komber d) leit dgg.

Daz im ir laster were leit $\mathrm{g}$. 
P.535,22 daz lat iu durch die frounen leit

23. $\sin \mathrm{D}$.

daz lat iu durch die froumen sin leit $\mathrm{g}$.

daz lat iu durch die frounen leit $\mathrm{Gad}$.

daz si iu Ggg. daz wirt iu $\mathrm{g}$.

P. 555, 7 so latz iu von mir niht swoere

8. $\sin \mathrm{D}$.

so latz iu von mir niht sin sware $\mathrm{g}$.

so latz iu von mir niht swaere d.

Lat ez iu (Lat iu iz gg) niht sin (wesen g. sein zuo g) sware Ggg

P.689,30 nu la dirz durch uns bede sin leit $\mathrm{Dg}$.

La dirz sin durch uns bede leit GGbgg.

nu si dirz d.

Wh.122, 2 nu la dir von mir niht so gach Kln.

nu (Doch p.) la dir von mir niht sein so gach mop.

la dir von mir niht wesen gach $\mathrm{t}$.

Tit.166, 4 Sigune, sueziu maget, la dir

sin die schrift an dem seile gar unmoere $\mathbf{G}$.

Süeziu magt Sigûne, là dir

die schrift der strängen sîn unmoere J.

Hierza füge ich zur vervollständigung des materials

P. 399, 6 und lazen in mit mir sin (wesen dgg) leit alle.

Lachmann schliesst sîn in klammern ein; dagegen folgt er an drei anderen stellen der überlieferung: P. 464,10 só lâ iu sîn mîn triegen leit. Wh. 1,10 la dîner tugende wesen leit. Wh. 342,10 là dir hiule wesen leit.

Ueberblicken wir die angefuhrten stellen, so finden wir die ellipse des verb. subst. bestätigt nur durch d P. 24, 18, wo aber eine verderbnis vorliegt, und $P .555,7$, durch $G^{2} d P .535,22$, endlich Wh. 122, 2 durch Kln. Nur an letzterer stelle würde an und fur sich betrachtet Lachmanns text als kritisch genügend beglaubigt gelten können, aber im zusammenhange mit den ubrigen analogen făllen wird man auch hier vorziehen müssen zu schreiben:

$$
\begin{aligned}
& \text { nu lâ dir von mir niht so gâch } \\
& \text { sîn, du enrîtes mit mir wider in. }
\end{aligned}
$$

An allen anderen stellen zwingt die thberlieferung zur beibehaltung von sin. Die urspringliche lesart hat, wie auch sonst, D am besten bewahrt. Die ubrigen handschriften suchten teils das metrum zu glätten, teils nahmen sie anstoss an der zerstuckelung des satzes durch den versschluss. Das aber ist bei Wolfram eine ganze gewöhnliche erscheinung. (Förster, Zur 
sprache und poesie Wolframs von Eschenbach, diss., Leipzig 1874, s. 3. Jander, Ueber metrik und stil in Wolframs Titurel, diss., Rostock 1883, s. 7.) Auch P. 129,16 wird mit D zu lesen sein (vgl. lesarten zu P. 142, 22)

der knappe sich dan al ein

huop zeime furte later wol getân.

und ebenso P. 570, 6

des kiule grœzer denne ein kruoc

was. er gienc gein Gâwâne her.

Lachmann lässt mit G. das verb. subst. fort; ähnlich Wh. 268, 9 mit l; auch hier kann man nur schwanken zwischen daz si ir weinen lieze sin verholen $\mathrm{Kn}$ und mit geringer abweichung mtz - oder daz si ir weinen lieze verholen | sîn: dâ solten etc. op. Die ellipse des verb. subst. nach lâzen war also Wolfram fremd. Er fügt im gegenteil bisweilen $\sin$ hinzu, wo wir es nicht erwarten, so P. 543, 25 f.

ich wil durch die herzogin

dich bî dem leben lâzen sîn.

Ich schliesse hieran die besprechung einiger anderer stellen. P. 69, 29-70,6 wird der bericht uber das turnier von Kanvoleis unterbrochen durch einen vorläufigen hinweis auf die bald darauf $(76,1 \mathrm{ff}$.) einzuführende gesantschaft der königin Ampflise:

$$
\begin{aligned}
& \text { Nu was oneh rois de Franze tôt, } \\
& \text { des wîp in dicke in grôze nôt } \\
& \text { brâhte mit ir minne: } \\
& \text { diu werde küneginne } \\
& \text { hete aldar nâch im gesant, } \\
& \text { ob er noch wider in daz lant } \\
& \text { wære }{ }^{1} \text { ) komen von der heidenschaft. } \\
& \text { des twanc si grôzer liebe kraft. }
\end{aligned}
$$

Lachmann hat den acht zeilen diese stelle angewiesen, während sie in den handschriften erst hinter 71,6 stehen. Zu der umstellung veranlasste inn offenbar der umstand, dass in der

1) Wenn ich in den citaten in einigen punkten vom Lachmannschen texte abweiche, so erlaube ich mir dafür auf meine kritischen bemerkungen zur metrik Wolframs von Eschenbach in der festschrift zur einweihung des Wilhelm-gymnasiums, Hamburg 1885, s. $55 \mathrm{ff}$. zu verweisen, wo diese abweichungen des weiteren begrindet sind. 
handschriftlichen uberlieferung die schilderung der rustung Gahmurets in zwei teile zerrissen wird. Aber durch diese umstellung sind keineswegs alle bedenken aus dem wege geräumt. In anderem zusammenhange macht Bötticher (Z. f. d. ph. XIII, 1882, s. 429) mit recht darauf aufmerksam, dass 70, 7

Ez wart dâ harte guot getân etc.

'der dichter fortfährt von dem turnier zu erzählen, als ob gar keine unterbrechung der darstellung stattgefunden hätte.' Nur übersieht er, wenn er diesen hinweis auf das verhältnis Gahmurets zur Anpflise als den ersten bezeichnet, dass auch P. 12, 4 ff.:

dô het der helt unverzagt

enpfangen durch liebe kraft

unt durch wîplîch geselleschaft

kleincetes tûsent marke wert.

swâ noch ein jude ptandes gert,

er möhtẹz derfür enpfâhen:

ez endorft im niht versmâhen.

daz sande im ein sin friundin.

einzig auf Anpflîse bezogen werden können (Bartsch z. d. st.). Ich lege gewicht auf den ausdruck kleinotes thent marke wert. Denn ebenso wird 71, $4 \mathrm{ff}$.

mir selben ich wol gunde

des er het an den lip gegert:

wand ez was maneger marke wert

die rtustung Gahmurets abgeschätzt. Und es ist mir wahrscheinlich, dass gerade die ähnlichkeit des ausdrucks, eines offenbaren notbehelfs, zu dem man noch P. 513, 22 vergleichen möge, den dichter vermöge einer eigentumlichen gedankenverbindung an Anpflîse erinnert und den einschub der in rede stehenden acht zeilen hervorgerufen hat. Man ist ja an manche sonderbarkeiten und gedankensprünge bei Wolfram gewöhnt, und wenn ich auch einen fall, der unserem genau entspräche, nicht beizubringen vermag, so wird man meine annahme doch wol gerade fur den anfang des gedichtes, wo es Wolfram besonders an ubung und gewantheit gebrach, nicht ganz abweisen können. Zunächst suchte der dichter wol nach einem reim auf drunde $i 1,3$, denn reimnot hat - das denke ich bei anderer gelegenbeit ausfuhrlicher nachzuweisen - noch mehr, als man bisher erkannt oder zugestanden hat, Wolframs aus- 
druck und darstellung bestimmt. Und indem er auf drunde : mir selben ich wol gunde reimte, wurde er weiter zu der sonderbaren umschreibung: des er het an den lîp gegert für die rtistung gefuhrt und so weiter zu dem ganzen excurs. Ich glaube daher, dass die zeilen in den handschriften an dem richtigen platze stehen.

P. 278, 11-18 wird das zelt der Cunnewâre von Lâlant beschrieben:

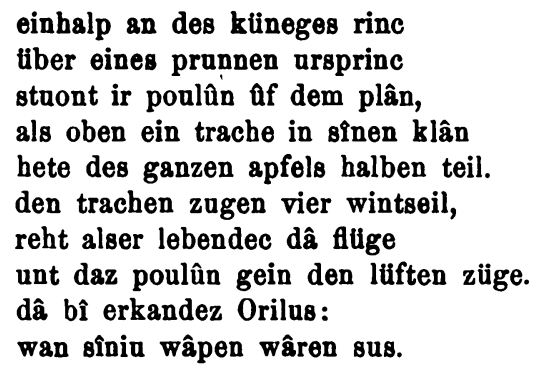

Za z. 14 gibt Bartsch die erklärung: 'als gebildet wie, anzusehen wie wenn. Auf der spitze des zeltes war ein halber apfel, den ein drache, das wappen der familie in den klauen hielt.' Hiermit widerspricht er sich selbst. Denn wenn wirklich eine apfelhälfte die zeltspitze gebildet hätte, eine annahme, deren unwahrscheinlichkeit sofort einleuchtet, wenn man sich nur ein solches zelt vergegenwärtigt, so wäre für die vergleichungspartikel als kein platz. Bartsch hat des dichters meinung nicht richtig erkannt. Wolfram vergleicht das aussehen des von einem drachen gekrōnten zeltes mit dem eines halben apfels, den ein drache in seinen klauen halte. Das tritt noch klarer hervor in der handschriftlichen uberlieferung. Denn statt als, wie Lachmann mit d schreibt, bieten die ubrigen handschriften als ez:

als ez oben ein trache in sînen klân hete, des ganzen apfels halben teil.

Man kann zweifeln, warum Lachmann hier, wie auch noch an einigen anderen stellen, von seinen sonstigen kritischen grundsätzen abgewichen ist. Vielleicht, dass ihm die volanstellung des $e z$, zu dem dann teil die apposition bildet, anstoss gab, aber so pleonastisch braucht Wolfram das pronomen der dritten person öfter: 
P. 272, 29 ff. ruochet ir nu høeren, wie Orilus des innen wart, avenliurę von Artûses vart? nach Bartsch' jedenfalls richtiger abteilung. P. 305,9 ff. vil volkes bot in werden gruoz, Gawane und dem ritter rôt. P. 353, 11 f. herberge namen sie, knappen, die dâ kômen hie. P. 429, 2 f. diu (8c. snert) wârẹn in undergangen, Gâwâns knappen an der (8o Gg. oder an des Dgg? ans Lachm.) strîtes stunt. P. 432, $17 \mathrm{ff}$. da ist Scherules: den sulen si biten des, geleites ze Dîanazdrûn (mit Bartsch). P. 589, 10 ff. $\hat{u} z$ Feire hie stuont enbor. P. 723, 17 ff. niht lieber möht ir sîn geschehn, wan daz si den künec solde sehn, Itonje, diu ouch dà saz. Wh. 12, $30 \mathrm{f}$. Gyburge süeze wart in sûr, den heiden und der kristenheit. Wh. 33, $30 \mathrm{f}$. des mohten si niht goberen, Die getouften, an der zit. Wh. 465, $20 \mathrm{ff}$. ich sol iu schaffen ề starke male die si tragen, künege die hie sint erslagen. Vgl. auch P. 233, 28 ff. viere die taveln legten of helfenbein vîz als ein snê, stollen die da komen $\hat{e}$.

Diese stellen stutzen die unsrige. Ein unterschied besteht allerdings darin, dass an den angezogenen stellen das pronomen demjenigen gegenstande, den es vertritt, pleonastisch vorausgeschickt und ihm dann dieser gegenstand selbst als apposition beigefugt wird, an unserer ein ihm verglichener, so dass das pronomen in der grammatischen form dem vorangehenden poulan entspricht, nicht dem folgenden teil. Eine derartige unmittelbare ubertragung ohne ein vermittelndes 'gleichsam, als, wie' entspricht aber durchaus dem sprachgebrauch und dem lebhaften geiste Wolframs (Kinzel, Zur charakteristik des Wolframschen stiles, diss., Halle 1873, s. 29, auch Z. f. d. ph. V. Bötticher, Ueber die eigentumlichkeiten der sprache Wolframs, diss., Wien 1876, s. 63, auch Germ. XXI). Sie ist hier um so mehr gerechtfertigt, weil ein doppeltes bild vorliegt, die vergleichung des zeltes seiner form nach mit einem halben apfel und sein aussehen vermöge der krönung durch einen drachen, als ob es von diesem in den klauen gehalten werde. Dieses bild wird auch in den folgenden zeilen

$$
\begin{aligned}
& \text { den trachen zugen vior wintseil, } \\
& \text { reht als er lebendec dâ flitge } \\
& \text { unt daz poulân gein den lutften zuige }
\end{aligned}
$$

noch fortgesetzt. Wolframs worte gestatten nicht die deutung, die ihnen A. Schultz (Das höfische leben zur zeit der minnesinger II, Leipzig 1880, s. 215) gibt, als ob auch hier der drache in getriebener arbeit auf einem goldenen knopf am first des zeltes angebracht gewesen sei. Wir haben ihn vielmehr mit 
ausgebreiteten flugeln unmittelbar uber dem zelt schwebend zu denken, 'als wenn er dasselbe in die luft entfuhren wolle', ähnlich, wie bei den zelten, die im Erec und Lanzelet beschrieben werden (Schultz a. a. 0. 317). Einige schwierigkeit machen allerdings die vintseil, von denen der drache gezogen sein soll; wintseil heissen sonst die seile, die die seitenwände des zeltes an am boden eingeschlagene pflöcke spannen (Schultz s. 216). Nun könnte man hier daran denken, wintseil von anderen seilen zu verstehen, die nur den drachen zu halten bestimmt gewesen wären. Jedoch dürte damit kaum das richtige getroffen sein, man wird vielmehr den unlogischen ausdruck Wolfiram hier wie anderwärts zu gute halten mulssen. Was endlich die form des poulûn betrifft, so ist Schultz' ansicht, dass es stets einen viereckigen grundriss gehabt habe, nicht genügend begrtundet. Wäre sie richtig, so würde der ohnehin geschmacklose vergleich des poulun mit einer apfelhälfte ganz unpassend werden, aber Wolfram schwebte jedenfalls eine runde form desselben vor. Vgl. P. 589,13 sinwel als ein gezelt ez was.

Ein starkes schwanken der handschriften zeigt sich P. 113, 23

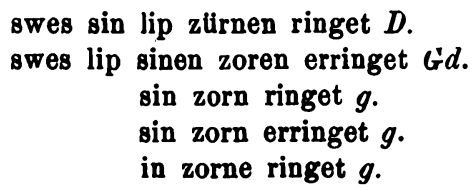

Lachmann kombiniert und schreibt:

swes lip sin zürnen ringet.

Bartsch erklärt ringen = gering anschlagen; und ebenso ubersetzt Lexer im Wtb.: 'wer sein (d. i. gottes) zurnen gering anschlägt, wem es gleichgultig ist'; aber das kann wol ringen uberhaupt nicht heissen. Alle im Mhd. wtb. und von Lexer beigebrachten stellen, den muot, die sware, last, trâren, leit, nôt, smerzen, pîn, arbeit, gemüete, zît, ungemach, vreude, laster, sorge, diu bant ringen, fuhren ubereinstimmend auf die bedeutung: leicht machen, erleichtern, abschwächen, besänftigen. So heisst auch P. 164, 4 lat iweren liden ringen es euren gliedern leicht machen. Dieser tatbestand, den Lachmann bei seinen hulfsmitteln noch nicht, wie wir, ubersehen konnte, macht seine combination unmöglich, so lange nicht aus ande- 
ren quellen ringen in der bedeutung 'gering anschlagen' belegt ist.

Auch in anderer hinsicht erweckt Lachmanns text bedenken. Die schilderung, wie Herzeloyde ihren neugeborenen sohn herzt, in ihm den verlorenen gemahl wider erstehen sieht und ihn selbst zu nähren beschliesst, gehört zu den schönsten und zartest empfundenen bei Wolfram. Herzeloyde beruft sich auf die gottesmutter (P. 113,17 ff.):

frou Herzeloyde sprach mit sinne:

'diu hœhste küniginne

Jêsus ir brüste bôt,

der sît durch uns vil scharpfen tôt

ame kriuze mennischlîche enpfienc

und sine triwe an uns begienc'.

Damit schliesst diese scene gut ab. Ein ganz mulssiger zusatz sind dagegen im munde der Herzeloyde die folgenden zeilen:

swes lîp sîn zürnen ringet,

des sêle unsamfte dinget,

swie kiuscher sî und wære.

des weiz ich wâriu mære.

Unangemessen ist, dass Herzeloyde so ihre kiusche selbst hervorhebt. Und die letzte zeile des weiz ich wâriu moere macht durchaus den eindruck zu einer nun folgenden erörterung tuberleiten zu sollen. Denn maere steht nie bei Wolfram, wie man hier annehmen musste, ganz farblos, sondern weist stets auf eine wirklich vorgetragene geschichte hin. Zu unserer stelle eine parallele bietet z. b. P. 271, 24

diu aventiure wert mære mich:

do Orilus der furste orkant elc.

nach Bartsch' interpunktion, die dem sinn besser entspricht, als die Lachmannsche.

Diese bedenken sprechen zugleich gegen den text der handschriften ausser $\mathrm{D}$, wenn vielleicht jemand daran denken sollte, diesen den vorzug zu geben. Ohnehin trăgt gerade D hier, wie Lachmann richtig erkannte, echt Wolframsches gepräge. Denn Wolfram hat eine vorliebe fur substantivierte infinitive; er scheint sie häufiger anzuwenden, als irgend ein anderer dichter, und ersetzte durch sie geradezu viele abstrakta, weil ihm die sinnliche verbalnatur viel mehr zusagte (Bötticher 
a. a. 0. 62). Das tritt freilich in den handschriften nicht immer rein hervor, sondern die schreiber haben häufig durch einsetzen der entsprechenden substantiva die eigenartigkeit des ausdrucks verwischt, wie z. b. die lesarten zu P. 89, 18. 91,30. 141,9. 234, 23. 259, 11. 26. 649, 4; Wh. 460, 20 beweisen mögen.

Demnach glaube ich, da es gilt von zwei ubeln das kleinere zu wählen, und da es mir wenigstens nicht gelungen ist eine in jeder beziehung befriedigende lösung zu finden, dass man ganz an D festzuhalten hat. Die vier zeilen würden alsdann nicht mehr zur rede der Herzeloyde gehören, sondern worte des dichters selbst sein und die passende uberleitung zu der weiteren erzählung bilden.

swes sin lip zürnen ringet, des sêle unsamfte dinget

wären zu erklären: wessen leib der zorn schwächt, dessen seele (nach Bartsch) erlangt schwer frieden. Die unflektierte form des pronomens, wenn man es nicht doch vorzieht ein aus abbreviatur der urhandschrift entstandenes versehen anzunehmen und sinẹn zu schreiben, wird geschutzt durch die reime P. 74, 2 . 75, 2. 707, 27. Befremdend ist der ausdruck zürnen für die trauer der königin um den verlust des gatten; aber nach dem Mhd. wtb. III, $905^{2}$ bedeutet zorn jede art plötzlich entstehenden unwillens, wie klein oder gross dieser sein mag. Hier war es Wolfram wol darum zu tun, Herzeloyde als mit dem schicksale hadernd darzustellen; darauf deuten auch ihre worte 109, $30 \mathrm{ff} .:$

hât got getriwe sinne,

sô lâzer mirn (sc. Parzival) ze frïhte komn.

ich hân doch schaden ze vil genomn

An minem stolzen werden man.

Noch auffälliger aber und daher wahrscheinlich auch der anlass, warum die ubrigen handschriften änderten, ist der pleonastische gebrauch des possessiven pronomens sin neben dem genetiv swes. Gr., Gr. IV, 351 bringt dafur uberhaupt nur ein mhd. beispiel. Etwas häufiger wird ir so pleonastisch verwandt. Die beispiele dafur bei Grimm und im Mhd. wtb. ergånzt Lucae, De nonnullis locis Wolframianis p. 3, anm. 2, wo indes Wh. 196, 27 missverstanden ist. Immerhin wird nach Grimms erörterungen und hinweis besonders auf das häufige 
vorkommen solchen redegebrauchs im volksmunde eine wendung, wie sie hier vorliegt, für Wolfram nicht als unmöglich betrachtet werden dürfen. Das bestätigt auch die Wolfram eigentumliche pleonastische vorsetzung des pronomens vor einen folgenden genitiv. Hierbei ist zwischen sin, ir und dem statt des possessivpronomens bei Wolfram häufig eintretenden genitiv des (Gr., Gr. IV, $341 \mathrm{ff}$.) in der anwendung kein unterschied zu merken.

P. 214, 5 ff. ine wil dich niht erlâzen, ir vater, Lîazen, dune bringẹst im dîne sicherheit. P. 273, 20 ff. mit nâhem umbevange behielt ir minne freuden prîs, der fürstîn und des fürsten wîs. P. 395, 12 f. ich wil gern ir kus mit gruoze hân, zweier frounẹn die ich hie sihe. P. 450, $9 \mathrm{ff}$. Parzival hôrte ir süezen wort, des vater, muoter und der kinde. Wh. $397,28 \mathrm{ff}$. die wol gezimierten ir brücke waren über bluotes furt, etslìchẹr $\hat{a z}$ Terramêrs geburt. - P. 189, $27 \mathrm{f}$. Sîn swester was diu muoter min, ivers wirtes. P. 325,7 ff. si jâhen daz her Gawân des kampfes sorge müese hân gein sîner wâren manheit, des fürsten der dâ vor in reit. P. 353, 28 f. sîn soumschrîn sint sô behuot, dînes ritters. P. 355,7 f. ob versnîden sol mîn swert sînen schilt, mînes hêrren wert. Wh. 270, 16 ff. ein zaher schiet den stoup von sinem claren vel, Rennewarts des knappen snel. Wh. 382,17 sîn schar, des künec Aropatîn. Wh. 425, 12 f. sîn bart was grâver danne der tuft, des alten künec Purrel. Wh. 433, 16 sînem vanen, des alten Heimrîch, ... wol gelanc. P. 662,16 f. Isajêsen si nande, des marschalc, Utepandragûn. Vgl. P. 392, 24 ff. do Gawan hete vernomen siniu wâpen, der mit in da strett.

Mit der verschiedenheit der lesarten P. 113, 23 möchte ich ausserdem das fehlen der zeilen 15 und 16 in $D$ in zusammen. hang bringen. Es ist mir wahrscheinlich, dass diese zeilen

si kêrtę sich niht an lôsheit:

diemuot was ir bereit

erst von einem schreiber eingeftugt wurden, als man mit änderung von zeile 23 diese und die drei folgenden zur rede der Herzeloyde gezogen hatte. Der schreiber mochte die entstehende unschicklichkeit selbst empfinden und suchte nun im voraus diese worte der Herzeloyde durch einen hinweis auf ihre demuitige, nicht leichtfertige gesinnung zu motivieren. Man mache nur den versuch und lese $113,1-14$ und $17-22$ hinter einander, und man wird empfinden, wie sehr das ganze durch die einfugung von z. 15.16 gelitten hat. Hierin wenigstens hoffe ich keinem widerspruch zu begegnen. Damit ist nun freilich nicht bewiesen, dass die zeilen nicht doch von Wolfram 
herruhren könnten, sondern interpoliert seien. Ueberhaupt ist bei dem eigenartigen, von dem gewōhnlichen nur zu hăufig abweichenden stile Wolframs der nachweis von interpolationen schwer zu erbringen; doch scheinen mir an einigen stellen sinn und uberlieferung auf annahme solcher hinzuflihren. Das wird mancher voraussichtlich obne weiteres von der hand weisen und Lachmanns 30-zeilen-gesetz dagegen anfuhren (Lachmann, vorrede IX; Haupt, Zs. f. d. a. XI, 49). Zwar hat schon San-Marte (Schulz, Ueber Wolfram von Eschenbachs rittergedicht Wilhelm von Orange, Quedlinburg und Leipzig 1871, s. 115 f.) begründeten einspruch gegen dasselbe erhoben, aber man scheint diesen einspruch geflissentlich $\mathrm{zu}$ ignorieren. Ich kann auf San-Martes ausführungen, denen ich völlig zustimme, verweisen und begnuge mich, da es in vielen fällen schwer gelingt uber etwaige interpolationen bei Wolfram zur klarheit durchzudringen, hier mit anfuhrung noch zweier stellen.

P. 654, 25. 26 fehlen Ddg, dagegen 23. 24 Gg. 23-26 hat nur g. Lachmanns berechnung, dass die vier verse auch in $\mathbf{F}$ gestanden haben sollten, bleibt unsicher. Die stelle (Gawans bote ist zurickgekehrt und hat ihm die nachricht gebracht, dass Artus seiner bitte willfahren und zu dem kampfe zwischen ibm und Gramoflanz mit seinem hofstaate kommen werde) lautet im zusammenhange v. $23 \mathrm{f}$. bei Lachmann:

Gâwâns sorge gar verswant:
niht wan freude er ime herzen vant.
Gâwân âz sorge in fröude trat.
den knappen erz verswîgen bat.
al siner sorge er gar vergaz,
er gienc hin wider unde saz,
und was mit freuden dâ ze hâs etc.

Hier spricht nun, sollte ich denken, wenn man die handschriftliche uberlieferung ansieht, alle wahrscheinlichkeit dafur, dass Wolfram nicht denselben gedanken, dass sich Gawans sorge in freude verwandelt, dreimal wird widerholt, sondern sich mit einmaliger widerholung begntigt haben, dass also nur entweder vers 23. 24 oder 25. 26, nicht aber alle vier echt sein können. Und zwar glaube ich, dass die in Ddg uberlieferten verse die echten sind. Der autor der in $\mathrm{Gg}$ sich findenden wollte wol die lästige widerholung durch einschiebung eines, der vorher- 
gehenden erzåhlung entnommenen zwischengedankens erträglicher machen.

P. 140, 1.2 ferner

du bist geborn von triuwen,

daz er dich sus kan riuwen.

fehlen in der klasse Dd. Dass diese verse von einem schreiber herrthren, , der, um in der geistreichen parallele meines namensvetters L. Bock (Wolframs von Eschenbach bilder und wörter von freude und leid, Strassburg $1879=$ Q. u. F., XXXIII s. 52) zu bleiben, das bedurfnis fuhlte auch einmal herz und schmerz zu reimen, glaube ich aus einer beobachtung des Wolframschen sprachgebrauches erhärten zu können. Wolfram gebraucht geborn oder erborn von nur im eigentlichen sinne der abstammung von einer person oder aus einem lande.

P. 9, 12 woerstu von Gylstram geborn. 54, 27 von Sibilje az der stat was geborn den er bat. 56,1 er ist erborn von Anschourve. 140, 26 ein Waleis von der muoter dîn bislu geborn von Kanvoleiz. 441,8 nie sô schœener lîp wart gelorn von menneschlicher fruht. 457, 16 nie kiuscher fruht von libe wart geborn. 474, 27 ich bin von einem man erborn, der mit tjost hât den lîp verlorn. 499, 13 von lther du bist er. born. 591, 6 si sint erborn von küneges art. 656, 15 von des nachkomn er ist erborn. 750, 24 sin wîp, von der ich wart geborn. 751, 29 wir han in ze rehter tjost verlorn, von dem wir bêde sîn erborn. 754, 19 liute, von den wir sîn erborn. 'Tit. 38, 1 Anphlisen wart ein kint gelâzen, erboren von fürsten künne. 58, 2 ich hore sagen, du sist erborn von der art, die nie kunde verdriezen etc. 128,2 er ist von den liuten erboren, die niht lant ir prîs nider sigen. 147,1 Si was von Kanadic erboren. Willeh. 30, 26 des toufes wer niht mîdet, sine snîde von den du bist erborn. 121, 21 diu zwei von den wir sin erborn. 131,1 Der was von ritters art erborn. 150, 21 wà nu die von mir sint erborn? 152, 22 die von Heimrich sint erborn. 167, 16 helde die von mir reborn waren unde ouch ich von in. 170,10 ob halt ein swacher (vgl. Paul, $\mathrm{Zn}$ Wolframs Willehalm in diesen Beitr. II, 324) garzûn von mime geslahte woere erborn. 255, 5 Tenabruns, erborn von Liwes Nugruns. 293, 11 bin ich von werder diet erborn. 318,11 die erborn sint von miner art. 338, 26 Pompeius, von des geslaht ich bin erborn. 358,16 daz ist der eltste sun mîn, von mînem èrsten wîbe erborn. 462, 25 durch die diu von iu ist erborn.

Wo es sich dagegen nicht um eigentliche abstammung handelt, sondern bern im ubertragenen sinne gebraucht wird, verbindet es Wolfram mit $a z$ : 
P. 659, $28 \mathrm{ff}$. swenne ich gedanke an mich nim, daz ich az freuden bin erborn, wirt freude noch an mir erkorn, da gît ein fruht die andern fruht. 732, $17 \mathrm{nu}$ bin ich doch $\hat{a z}$ minne erborn : wie hân ich minne alsus verlorn? 738, 21 dise zwêne wârẹn uz krache erborn. 763, $20 \mathrm{der}$ was az rehtem prîs erborn. Tit. 35,2 si wâręn az lâterlîcher minne erborn.

Die erklärungen, die Bartsch zu diesen stellen gibt, treffen zwar den sinn, verwischen aber die eigenart der hier zu grunde liegenden anschauung. Wir haben in diesen wendungen offenbar eine sehr kuhne personification zu sehen, so dass die eigenschaften oder tătigkeiten (krach P. 738, 21 von der speer- und schildbrechenden tätigkeit des ritters in parallele mit dem galm des löwen) als erzeuger von menschen gedacht, bei unserem dichter sogar wie die menschen geschlechter bilden. P. 680, 2 f. $\hat{u} z$ der tjoste geslehte wârẹn si bêde samt erborn; denn $\hat{u} z$ der tjoste geslehte steht nicht anders, als oben von küneges art, von Pompeius geslehte etc. Bartsch' anmerkung: 'aus einem geschlecht, welches sich auf tjostieren verstand' umschreibt nur den sinn der stelle, erklärt den ausdruck nicht. In übertragenem sinne von ebenbürtigkeit der abstammung, nicht von eigentlicher verwantschaft ist auch zu verstehen Wh. 291,27 ff. ir (sc. Kyburcs) herze spehte rehte, daz er (sc. Rennewart) az ir geslehte endeliche ware erborn, swie er halt danne ware verlorn. Endlich lesen wir, den versen, von denen wir ausgiengen, auch dem gedanken und wortlaut nach entsprechend Wh. 298, $18 \mathrm{ff}$. sî wir reborn $\hat{u z}$ trive ganz, die zehen lêret missewende mîn armeclîch ellende.

Diese etwas längere auseinandersetzung, bei der ich hoffentlich keine in frage kommende stelle ubersehen habe, schien geboten, um P. 140,1.2 als mit dem Wolframschen sprachgebrauch unvereinbar zu erweisen. Die verse sind ohnehin unpassend und uberflussig. Denn da Sigûne noch nicht weiss, dass Parzival ihr verwanter ist, kann sie von triuwe, die er hier bewähren soll, nicht wol reden. Vielmehr enthalten die vorangehenden zeilen

si sprach zem knappen: 'du hâst tugent.

gêret sî dîn stieziu jugent

unt dîn antlütze minneclîch. 
deiswar du wirst noch sælden rich.

disen ritter meit dez gabylôt,

er lac ze tjostieren tôt

die ansreichendste antwort auf Parzivals teilnehmende frage nach dem toten Schîanatulander und auf sein erbieten, denselben rächen zu wollen. Der zusatz kann nur störend wirken.

HAMBURG.

C. BOCK. 